Article

\title{
Acetamiprid Affects Destruxins Production but Its Accumulation in Metarhizium sp. Spores Increases Infection Ability of Fungi
}

\author{
Monika Nowak ${ }^{1}$, , Przemysław Bernat ${ }^{1}$, Julia Mrozińska ${ }^{2}$ and Sylwia Różalska ${ }^{1, *([)}$ \\ 1 Department of Industrial Microbiology and Biotechnology, Faculty of Biology and Environmental Protection, \\ University of Łódź, 90-237 Łódź, Poland; monika.nowak@unilodz.eu (M.N.); \\ przemyslaw.bernat@biol.uni.lodz.pl (P.B.) \\ 2 Scientific Students Group "SKN Bio-Mik", Faculty of Biology and Environmental Protection, University of \\ Łódź, 90-237 Łódź, Poland; julia.mrozia@gmail.com \\ * Correspondence: sylwia.rozalska@biol.uni.lodz.pl
}

Received: 10 August 2020; Accepted: 5 September 2020; Published: 11 September 2020

check for updates

\begin{abstract}
Metarhizium sp. are entomopathogenic fungi that inhabit the soil environment. Together, they act as natural pest control factors. In the natural environment, they come into contact with various anthropogenic pollutants, and sometimes, they are used together and interchangeably with chemical insecticides (e.g., neonicotinoids) for pest control. In most cases, the compatibility of entomopathogens with insecticides has been determined; however, the influence of these compounds on the metabolism of entomopathogenic fungi has not yet been studied. Secondary metabolites are very important factors that influence the fitness of the producers, playing important roles in the ability of these pathogens to successfully parasitize insects. In this study, for the first time, we focus on whether the insecticide present in the fungal growth environment affects secondary metabolism in fungi. The research revealed that acetamiprid at concentrations from 5 to $50 \mathrm{mg} \mathrm{L}^{-1}$ did not inhibit the growth of all tested Metarhizium sp.; however, it reduced the level of 19 produced destruxins in direct proportion to the dosage used. Furthermore, it was shown that acetamiprid accumulates not only in plant or animal tissues, but also in fungal cells. Despite the negative impact of acetamiprid on secondary metabolism, it was proofed to accumulate in Metarhizium spores, which appeared to have a stronger infectious potential against mealworm Tenebrio molitor, in comparison to the insecticide or the biological agent alone.
\end{abstract}

Keywords: entomopathogens; mycoinsecticides; secondary metabolites; insect pathogenesis; acetamiprid accumulation

Key Contribution: Acetamiprid represses destruxins production by Metarhizium sp.; Spores of M. brunneum with accumulated acetamiprid have increased infectivity against $T$. molitor.

\section{Introduction}

Synthetic insecticides are important pesticides for both agricultural and domestic pest control. Among them, neonicotinoids (e.g., acetamiprid, imidacloprid, thiacloprid) are the most extensively used worldwide because of their high effectiveness in controlling crop and domestic pests [1]. They account for more than $25 \%$ of the global insecticide market and are now being considered as a replacement for many existing conventional insecticide classes [2]. Neonicotinoids' popularity is largely due to their physicochemical properties, high effectiveness, low resistance, and the fact that they are less harmful to mammals compared to other insecticides. For example, to protect fruit plants against pests, up to $3 \mathrm{~g}$ per $100 \mathrm{~m}^{2}$ of a popular pest control product (e.g., Mospilan $20 \mathrm{SP}$ ) is used with acetamiprid as the 
active substance are used and, as a consequence, acetamiprid penetrates the soil and bioaccumulates. According to data from the European Food Safety Authority (EFSA), ${ }^{14}$ C-acetamiprid was identified as the major constituent of the radioactive residues in all plant parts (the study included eggplants, apples, carrots and cabbage) in an amount of 30-90\% within 14-90 days after the last application [3]. The ability of neonicotinoids to accumulate in plants is known to increase the probability of environmental contamination and exposure to nontarget organisms [3-5]. In addition, despite the lack of recognition of acetamiprid as a compound persisting in soil, its degradation in environmental conditions has been found to last up to 43 days [3].

Among all methods of insect control, biological methods deserve special attention. Metarhizium sp., the common insect pathogens in wildlife, are very efficient bioinsecticides usually applied in practice [6] Their effectiveness against insects depends mainly on their infective potential, i.e., the ability to produce extracellular lytic enzymes and secondary metabolites [7,8]. While the extracellular enzymes are well studied, equally important secondary metabolites, which play critical roles in the ability of Metarhizium to successfully parasitize their hosts and ultimately contribute to the success or failure of these fungi as biological control agents, are quite often neglected [9]. Entomopathogenic fungi produce a variety of bioactive metabolites including $>40$ cyclic hexadepsipeptides destruxins (dtxs) [8]. It is suggested that in attacked insects, dtxs induce paralysis and muscle contraction via muscle depolarization by the direct opening of $\mathrm{Ca}^{2+}$ channels in the membrane $[10,11]$. Besides their insecticidal activity, dtxs have also potential as pharmaceuticals showing antiviral, antitumor, cytotoxic, immunosuppressant or antiproliferative effects [12,13]. However, due to the endophytic properties of Metarhizium sp., the presence of dtxs can be found in, e.g., potatoes [14], maize and strawberries [15], causing dtxs to enter the food chain and pose a threat to human health.

The aim of this work was to determine the influence of acetamiprid on the growth and the secondary metabolism of Metarhizium, which is considered as a significant factor during the pest infection process. Furthermore, we checked whether acetamiprid could be accumulated by Metarhizium, how it affected the production of dtxs, and whether it affected the ability of fungi to infect insects. This study could help to understand the potential risks of a harmful influence of acetamiprid on soil-inhabiting fungi and their infectious potential, which plays an important role in maintaining the ecological balance.

\section{Results}

\subsection{Fungal Biomass Yield of Metarhizium sp. in the Presence of Acetamiprid}

Among the tested strains, M. anisopliae and M. robertsii IM2358 were the best growing species, while $M$. brunneum and $M$. robertsii ARSEF727 grew at a similar medium rate (biomass yield about $\left.6 \mathrm{~g} \mathrm{~L}^{-1}\right)$. The slowest growing species was M. globosum. It turned out that acetamiprid did not inhibit growth in any of the tested strains, even at the highest concentration of $50 \mathrm{mg} \mathrm{L}^{-1}(p>0.05)$ (Figure 1). Literature data also provides information that acetamiprid has no harmful effect on conidia germination and production or vegetative growth in M. anisopliae —strain E9 (ESALQ/USP) [16]. The lack of toxic effect was also confirmed in the presented study.

\subsection{Quantitative Analyses of the Content of Acetamiprid in Metarhizium Fungal Cultures}

In this study, the fungal ability to eliminate acetamiprid added to cultures at concentrations of 5, 25 and $50 \mathrm{mg} \mathrm{L}^{-1}$ was verified (Figure S1). None of the tested Metarhizium sp. showed the highly effective ability to remove acetamiprid from the Czapek Dox culture medium after seven days of incubation. At the concentration of $5 \mathrm{mg} \mathrm{L}^{-1}$, a slight elimination capacity was demonstrated for $M$. brunneum and $M$. robertsii IM6519 (the average removal rate reached 29 and 24\%, respectively). In a situation where five times more acetamiprid was added to the fungal culture, a loss was observed in the samples of all tested strains. M. anisopliae, M. globosum and M. brunneum removed respectively 31, 25 and $24 \%$ of acetamiprid from the culture medium. In the case of the other species, the substrate 
elimination was below 20\%. In the fungal cultures, where the insecticide concentration was $50 \mathrm{mg} \mathrm{L}^{-1}$ there was no loss of more than $20 \%$ of the insecticide for any species. However, studies of the content of acetamiprid separately in the mycelium and culture medium showed that it was accumulated in the fungal cells.

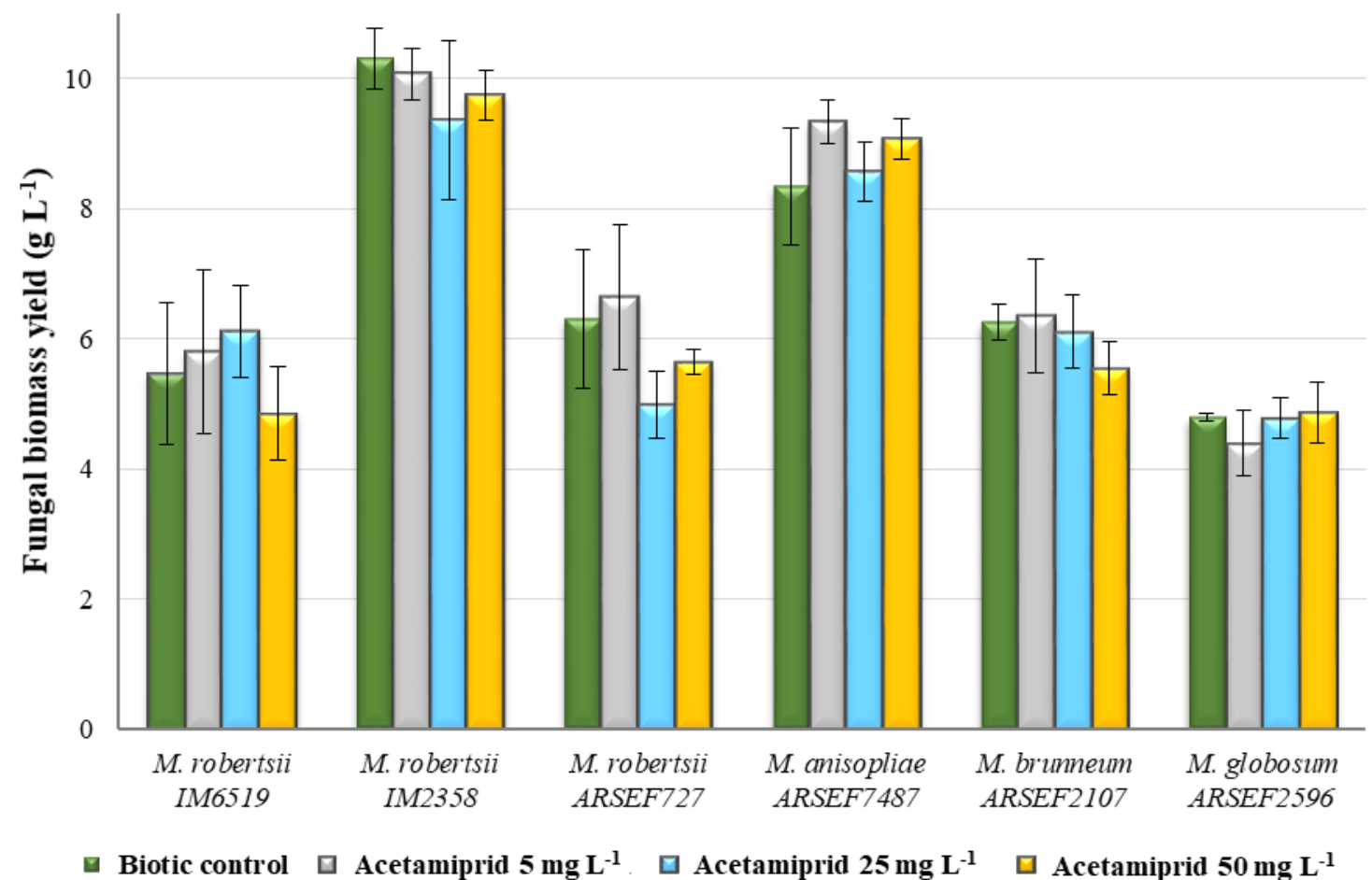

Figure 1. Influence of acetamiprid at concentrations of 5,25 and $50 \mathrm{mg} \mathrm{L}^{-1}$ on fungal biomass yield of Metarhizium sp. One-way ANOVA was used for investigations of statistical significance. All differences are statistically insignificant $(p>0.05)$.

This insecticide has proved the ability to accumulate, inter alia, in the tissues of plants where, through translocation, it can even move from the roots to the shoots $[17,18]$. It was found that due to its good solubility in water, acetamiprid has a strong toxic effect on aquatic organisms where it bioaccumulates by sorption mechanisms characteristic for compounds with high polarity [19,20]. Although acetamiprid accumulation ability has been described for several different species, in this paper, we present for the first time that entomopathogens, which are often used interchangeably or alternatively with various insecticides in agriculture, can also accumulate this compound, without metabolizing it inside the cells (during seven days of incubation). Herein, the amount of acetamiprid on the mycelium dry weight $\left(\mathrm{mg} \mathrm{g}^{-1}\right)$ was directly proportional to the increasing concentration $(p<0.05)$ (Figure 2). This trend was similar for each tested species while M. brunneum exhibited the highest accumulation potential. The rest of the strains showed statistically significant differences in the amounts of acetamiprid accumulated in the cells at the three concentrations used. At the concentration of $25 \mathrm{mg} \mathrm{L}^{-1}$ in $M$. anisopliae the highest amount of the accumulated insecticide $(0.69 \mathrm{mg}$ per $\mathrm{g}$ of dry weight) was observed ( $p<0.05$ ), but it is worth noting that this species had been previously found to have high biomass yield (Figure 1). M. robertsii IM6519, M. robertsii IM2358 and M. globosum accumulated in the cells' comparable amounts of acetamiprid (approximately 0.36-0.45 mg per $\mathrm{g}$ of dry weight, $p>0.05$ ). By comparison, $0.12 \mathrm{mg}$ per $\mathrm{g}$ of dry weight was found in M. brunneum $(p<0.05)$. The situation changed for the acetamiprid concentration of $50 \mathrm{mg} \mathrm{L}^{-1}$ in the fungal culture and the amount of the toxic substrate accumulated in the mycelium leveled out (Figure 2). The largest quantity was detected for M. globosum (1.05 mg per $\mathrm{g}$ of dry weight), which was different from the other 
species (except $M$. anisopliae, $p>0.05$ ). In all $M$. robertsii strains and the $M$. anisopliae strain studied, the acetamiprid amounts determined per $g$ of dry weight were comparable.

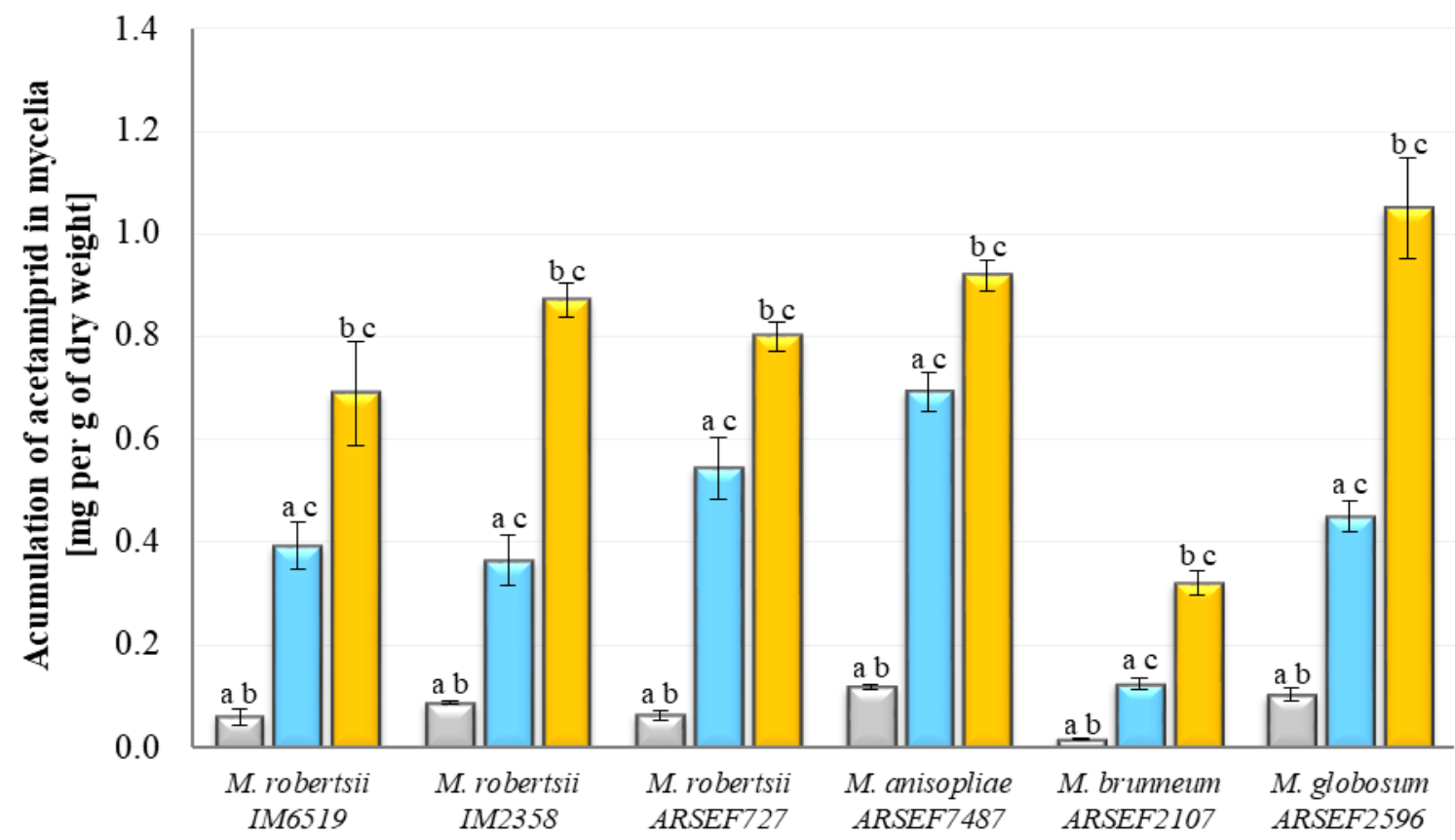

$\square$ Acetamiprid $5 \mathrm{mg} \mathrm{L}^{-1} \square$ Acetamiprid $25 \mathrm{mg} \mathrm{L}^{-1} \quad \square$ Acetamiprid $50 \mathrm{mg} \mathrm{L}^{-1}$

Figure 2. Accumulation of acetamiprid at concentrations of 5, 25 and $50 \mathrm{mg} \mathrm{L}^{-1}$ in the mycelium of Metarhizium sp. One-way ANOVA and Tukey's test were used for investigations of statistical significance. $(\mathbf{a}-\mathbf{c}) p<0.05$. Statistically significant differences between samples at individual concentrations within the species. (a) Between samples with acetamiprid at concentrations of 5 and $25 \mathrm{mg} \mathrm{L}^{-1}$; (b) between samples with acetamiprid at concentrations of 5 and $50 \mathrm{mg} \mathrm{L}^{-1}$; (c) between samples with acetamiprid at concentrations of 25 and $50 \mathrm{mg} \mathrm{L}^{-1}$.

\subsection{Analyses of Destruxins in Fungal Cultures of Metarhizium sp.}

According to literature data, M. anisopliae, M. robertsii and M. brunneum species have genes responsible for the production of dtxs [8]. Dtxs in M. globosum were also marked in this work, but their content was very low compared to the other species (in the order of 0.002 and $0.004 \mathrm{mg} \mathrm{L}^{-1}$ for $\mathrm{dtx} \mathrm{A}$ and $\mathrm{dtx} B$, respectively), so this species was excluded from further analyses. M. robertsii ARSEF727 had the lowest content of dtxs in the biotic control compared to the other species. The expression of genes responsible for the production of specific units making up the dtxs structure might not be as high as in the case of the other strains [8]. It cannot be said that the low concentrations of dtxs in $M$. robertsii ARSEF727 and M. globosum were caused by the poor growth of these fungi, because their growth rate was similar to that of M. brunneum and M. robertsii IM6519, which turned out to be the species with the highest content of dtxs in the biotic controls. Interestingly, M. anisopliae, in which the synthesis of dtxs has been accurately described, did not turn out to be the best producer [21]. M. robertsii IM2358 was also found to have higher levels of dtxs than M. anisopliae.

According to literature data, none of the fungal species has the capacity to produce all 39 types of dtxs, but $M$. anisopliae produces the majority of them [8]. In all tested strains, except M. globosum, 19 dtxs were determined. Due to the lack of chromatography standards, accurate quantitative analyses were performed only for $\mathrm{dtx} \mathrm{A}$ and $\mathrm{B}$. Therefore, the amounts of $\mathrm{dtx} \mathrm{A}$ and $\mathrm{B}$ and the other types were described separately. It is worth noting that $\operatorname{dtx} A$ and $d t x B$ are the main metabolites and occur in higher concentrations compared to other dtxs [22], which was also confirmed in this work. 
It was checked whether, despite the lack of the influence of acetamiprid on the growth of the tested fungi, this insecticide affected the secondary metabolism. As mentioned above, three concentrations of acetamiprid $\left(5,25\right.$ and $\left.50 \mathrm{mg} \mathrm{L}^{-1}\right)$ were examined. It turned out that the lowest concentration caused disturbances in the synthesis of the secondary metabolites of Metarhizium $(p<0.05)$. The use of higher doses of acetamiprid contributed to a gradual reduction in the amount of detected dtxs. Declines in the content of dtxs A and B were quite proportional (Figure 3). For M. brunneum there were no statistically significant differences between the amounts of $\mathrm{dtx} A$ at concentrations of 25 and $50 \mathrm{mg} \mathrm{L}^{-1}$. The differences between all the concentrations used, which were determined for $\mathrm{dtx} B$ were statistically significant. The amounts of $\mathrm{dtx}$ A for $M$. robertsii IM2358 did not differ significantly between the concentrations of 5 and $25 \mathrm{mg} \mathrm{L}^{-1}$, and for $\mathrm{dtx} B$ between the concentrations of 25 and $50 \mathrm{mg} \mathrm{L}^{-1}$. No statistically significant differences between the concentrations of 25 and $50 \mathrm{mg} \mathrm{L}^{-1}$ for $\mathrm{dtx} \mathrm{A}$ and $\mathrm{B}$ were found for $M$. robertsii ARSEF727.

The most harmful effect of acetamiprid on the production of dtxs was noted for M. brunneum, despite the fact that the growth rate and amounts of $d t x s$ in the biotic sample were similar to those observed for $M$. robertsii IM6519. At the concentration of $5 \mathrm{mg} \mathrm{L}^{-1}$, the contents of dtxs A and B were 56.43 and $41.93 \%$ lower than in the biotic controls, respectively. The highest dose of the insecticide resulted in a very large reduction in the contents of dtxs A and B to 11.99 and $14.58 \%$ of the biotic control, respectively. As mentioned above, $M$. brunneum accumulated in the mycelium the lowest quantities of acetamiprid per $g$ of dry weight (Figure 2). It seems that $M$. brunneum defended itself against the presence of the insecticide in the mycelium. This could have been the reason why acetamiprid so heavily influenced the contents of dtxs in this strain. Dtxs production in M. robertsii ARSEF727 and M. robertsii IM6519 was also inhibited at the highest concentration of the insecticide and for dtx A the amounts were 90.99 and $72.09 \%$ lower than in the biotic control, and for $\mathrm{dtx} \mathrm{B} 82.53$ and $73.15 \%$, respectively (Figure 3).

Comparable decreases in the contents of $\mathrm{dtxs}$ A and B were observed for M. anisopliae and M. robertsii IM2358 strains. With the increase in the acetamiprid concentration, the amounts of dtx A were lower by $10.04,17.33$ and $43.05 \%$ for $M$. robertsii IM2358 and by $13.10,33.24$ and $43.61 \%$ for M. anisopliae. It was noticed that the dose of $25 \mathrm{mg} \mathrm{L}^{-1}$ was more toxic to M. anisopliae than to M. robertsii IM2358. The concentration of $\mathrm{dtx} B$ for $M$. anisopliae decreased in a similar way to the content of $\mathrm{dtx} A$ $(9.49,33.05$ and $40.03 \%$ less than in the biotic control, with the increase in the acetamiprid concentration). For M. robertsii IM2358 at the of concentrations 5 and $25 \mathrm{mg} \mathrm{L}^{-1}$ the decreases were similar to dtx A (7.94 and 19.68\% less than in the biotic control, respectively), while at the concentration of $50 \mathrm{mg} \mathrm{L}^{-1}$, the content of $\mathrm{dtx}$ B decreased by $24.21 \%$ (the reduction was almost two-fold smaller than for $\mathrm{dtx} \mathrm{A}$ ).

The levels of the other $17 \mathrm{dtxs}$, for which chromatographic standards are not available, were estimated based on the chromatographic peak areas. It turned out that the tested species differed in terms of the profile of dtxs, which was confirmed by the PCA (Figure 4). To the best of our knowledge, this kind of analysis had never been done before.

A similar dtxs profile was obtained for M. anisopliae and M. robertsii IM2358 (Figure 4). These strains differed from the others due to the high levels of $\mathrm{dtx}$ B1 and dtx Ed. A superior decrease in the levels of these $\mathrm{dtxs}$ and $\mathrm{dtx} \mathrm{D}$ was observed for $M$. robertsii IM2358 with the acetamiprid concentration of $50 \mathrm{mg} \mathrm{L}^{-1}$, hence in the PCA chart, this tested sample was distinguished and shown to migrate towards M. robertsii IM6519 (tested samples with acetamiprid at concentrations of 5 and $25 \mathrm{mg} \mathrm{L}^{-1}$ ), in which the levels of these $\mathrm{dtxs}$ were also low (Figure 4). The level of $\mathrm{dtx}$ DesmA distinguished M. anisopliae from M. robertsii IM2358, as lower values were obtained for $M$. anisopliae. The level of synthesized dtx A1 was definitely a factor differentiating $M$. anisopliae from the other tested species, because for this strain, the highest level of $\mathrm{dtx}$ A1 was determined. Such close proximity of M. anisopliae and M. robertsii IM2358 on the PCA chart was in line with the presented previously. These species were characterized by a similar growth (Figure 1) and the effect of acetamiprid at concentrations of 5,25 and $50 \mathrm{mg} \mathrm{L}^{-1}$ on the decrease in the amounts of dtxs A and B (Figure 3). 


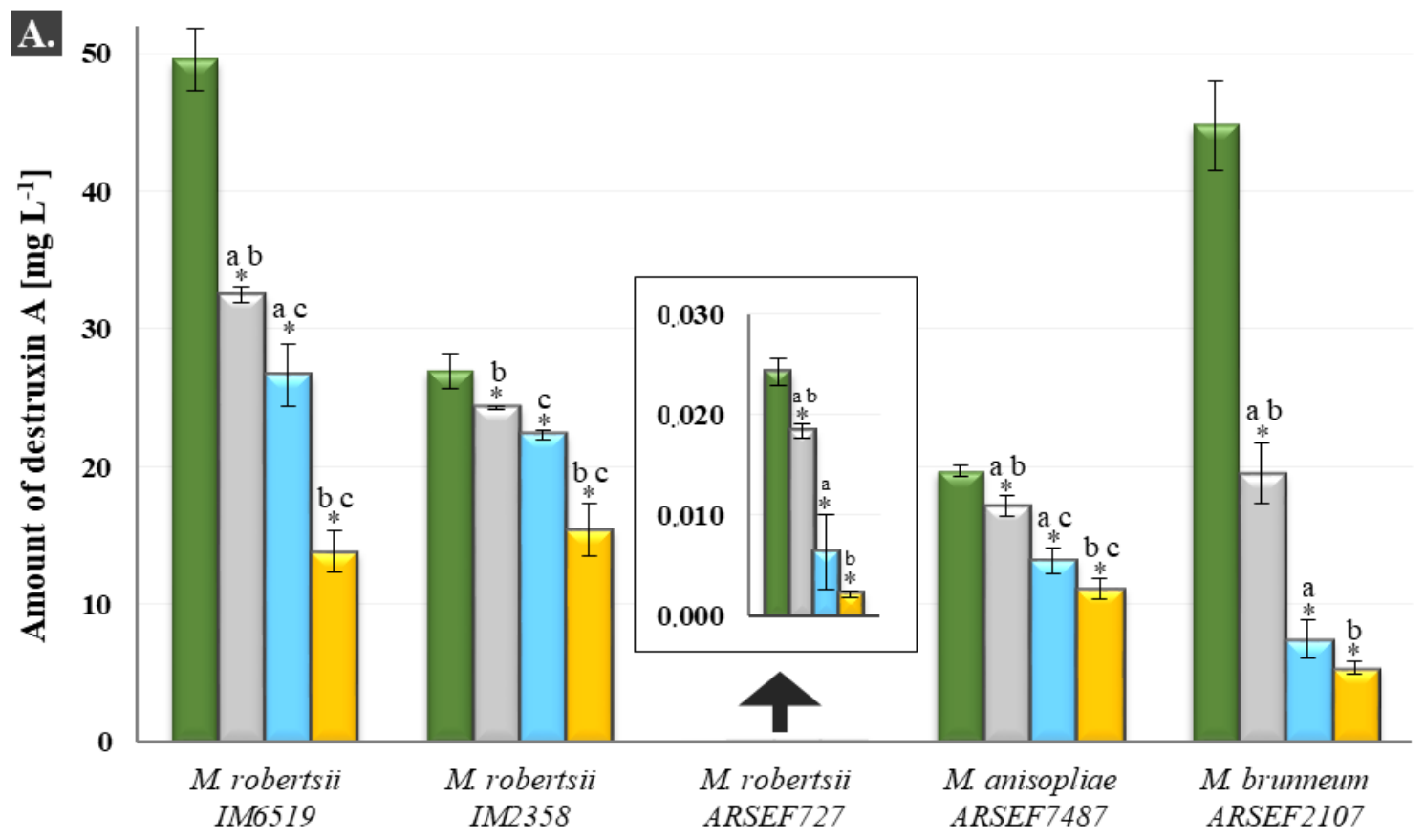

$\square$ Biotic control $\square$ Acetamiprid $5 \mathrm{mg} \mathrm{L}^{-1}$. $\square$ Acetamiprid $25 \mathrm{mg} \mathrm{L}^{-1} \square$ Acetamiprid $50 \mathrm{mg} \mathrm{L}^{-1}$

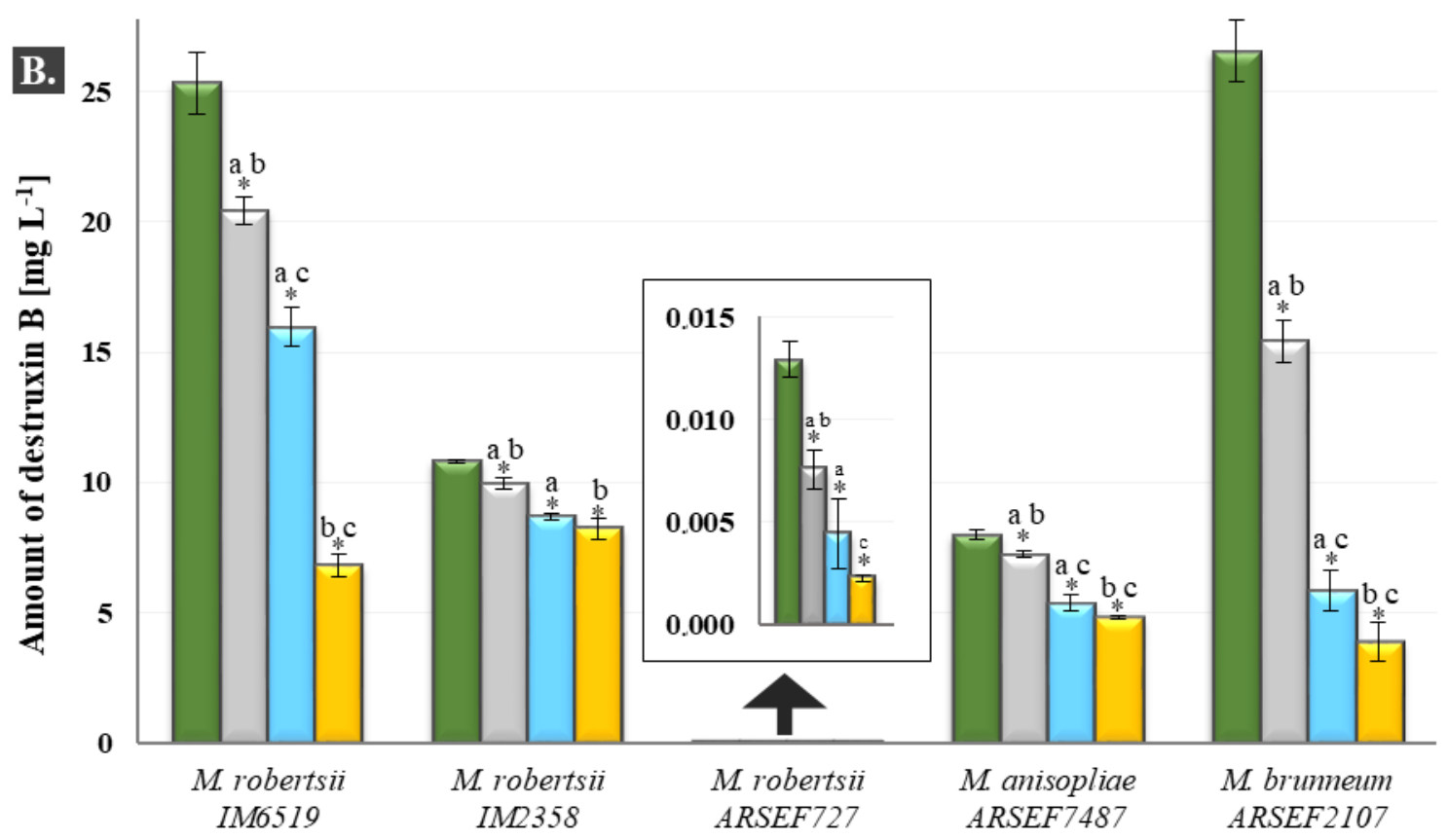

$\square$ Biotic control $\square$ Acetamiprid $5 \mathrm{mg} \mathrm{L}^{-1} \square$ Acetamiprid $25 \mathrm{mg} \mathrm{L}^{-1} \square$ Acetamiprid $50 \mathrm{mg} \mathrm{L}^{-1}$

Figure 3. Effect of acetamiprid at concentrations of 5, 25 and $50 \mathrm{mg} \mathrm{L}^{-1}$ on the amounts of destruxins A (A) and B (B) produced by Metarhizium species. One-way ANOVA and Tukey's test were used for investigations of statistical significance. ${ }^{*} p<0.05$. Statistically significant differences between samples with acetamiprid at concentrations of 5,25 and $50 \mathrm{mg} \mathrm{L}^{-1}$ and their biotic controls within the species; (a-c) $p<0.05$-statistically significant differences between samples at individual concentrations within the species. (a) Between samples with acetamiprid at concentrations of 5 and $25 \mathrm{mg} \mathrm{L}^{-1}$; (b) between samples with acetamiprid at concentrations of 5 and $50 \mathrm{mg} \mathrm{L}^{-1}$; (c) between samples with acetamiprid at concentrations of 25 and $50 \mathrm{mg} \mathrm{L}^{-1}$. 

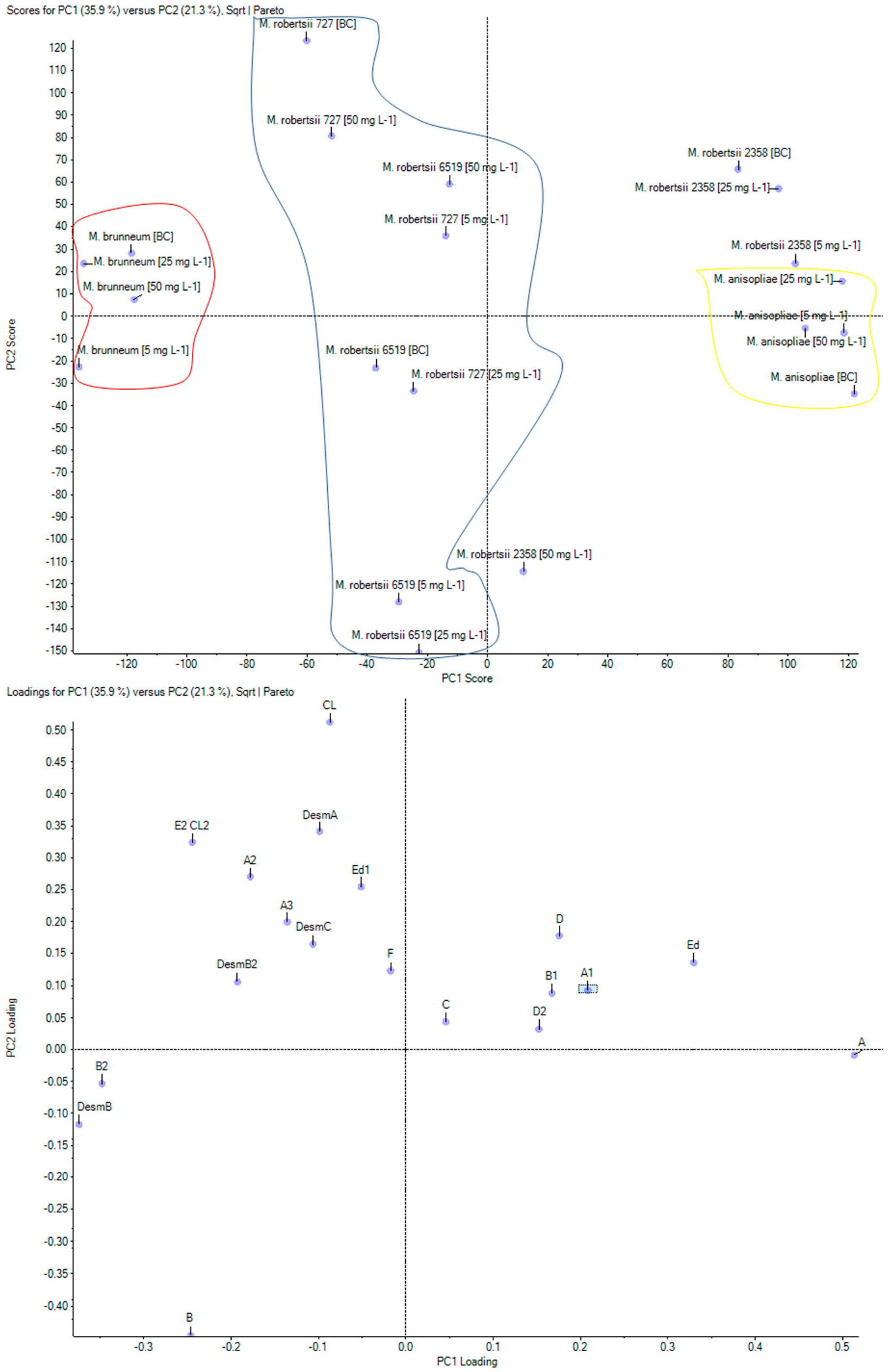

Figure 4. Results of principal component analysis (PCA) on the profile of destruxins of Metarhizium species in samples without the addition of acetamiprid and with acetamiprid at concentrations of 5, 25 and $50 \mathrm{mg} \mathrm{L}^{-1}$. PC1 against PC2 scores chart (top); PC1 against PC2 loadings chart (bottom). 
The above-mentioned results of the accumulation of acetamiprid in the mycelium showed that M. brunneum differed from the other species because of the lowest quantity of the insecticide bound in the fungal cells (Figure 2). It turned out that the dtxs profile of this strain also contributed to its differentiation, particularly to the high levels of dtxs DesmB, B2, DesmB2 and E2CL2 and the relatively low levels of dtxs Ed and D2.

Analysis of the PCA chart was more complicated for the strains M. robertsii ARSEF727 and M. robertsii IM6519. Their profiles were quite similar; however, it was possible to see differences in the contents of individual dtxs (Figure 4). The differentiating factor for M. robertsii ARSEF727 was the high level of dtx Ed1, particularly for the sample with acetamiprid at the concentration of $50 \mathrm{mg} \mathrm{L}^{-1}$, where the addition of acetamiprid did not reduce the level of dtx Ed1 more than in the sample with the addition of acetamiprid at the concentration of $25 \mathrm{mg} \mathrm{L}^{-1}$. The high level of dtx E2CL2 slightly differentiated from the biotic sample with $M$. robertsii ARSEF727 from the other samples of this species. Dtx CL was at a high level in M. robertsii IM6519, but in the sample with acetamiprid at a concentration of $50 \mathrm{mg} \mathrm{L}^{-1}$, it definitely decreased. The close proximity of $M$. robertsii IM6519 (the biotic control and the sample with acetamiprid at a concentration of $50 \mathrm{mg} \mathrm{L}^{-1}$ ) to $M$. robertsii ARSEF727 was due to the high level of $d t x$ A3. However, the low level of this $d t x$ in the tested sample with acetamiprid at a concentration of $25 \mathrm{mg} \mathrm{L}^{-1}$ in $M$. robertsii ARSEF727 made this strain more similar to M. robertsii IM6519 (samples with acetamiprid at concentrations of 5 and $25 \mathrm{mg} \mathrm{L}^{-1}$ ).

\subsection{Permeability of the Cell Membrane and the Content of Acetamiprid in Spores and Subcellular Fractions of the M. brunneum}

M. brunneum was chosen to conduct an experiment on the effect of acetamiprid on the permeability of biological membranes, due to the greatest inhibition of dtxs production by this compound, even to $88 \%$ at the concentration of $50 \mathrm{mg} \mathrm{L}^{-1}$ (Figure 3). Dtxs are extracellular metabolites; however, their synthesis takes place in fungal cells [23]. The properties of the cell membrane, including permeability, potential and fluidity, have an influence on the cell secretion process [24]. Acetamiprid did not reduce the permeability of biological membranes at any of the used concentrations $(p>0.05)$. The reduced amount of dtxs determined in the samples with the addition of the toxic insecticide was not associated with a disorder of the membrane permeability system (Table S1).

Due to the high inhibition of dtxs production, it was checked whether acetamiprid accumulated in the spores of M. brunneum. Additionally, its amount in the cell wall and other subcellular structures of the fungus was determined. It turned out that acetamiprid accumulated in the spores $\left(0.280 \pm 0.05 \mu \mathrm{g}\right.$ per $10^{6}$ of spores), while in cell fractions almost 6 -fold more acetamiprid was detected in the cell wall $(27.61 \pm 3.75 \mu \mathrm{g}$ per $\mathrm{g}$ of dry weight) than in the other subcellular structures $(4.78 \pm 0.65 \mu \mathrm{g}$ per $\mathrm{g}$ of dry weight).

\subsection{Influence of Acetamiprid, Spores of M. brunneum ARSEF2107 and the Combination of Spores and Acetamiprid on the Mortality of Tenebrio molitor (Mealworm)}

Acetamiprid is used worldwide as an effective insect control agent. The dose of acetamiprid at used in these studies $\left(5,25\right.$ and $50 \mathrm{mg} \mathrm{L}^{-1}$ ) caused mortality of T. molitor (Figure 5).

Additionally, the action of the neonicotinoid was compared to the killing properties of M. brunneum spores. The obtained results of acetamiprid accumulation in the fungal spores prompted us to determine what effect could be generated by the combination of M. brunneum spores with the accumulated acetamiprid and whether such a mixture could be an alternative to using the chemical insecticide alone. The results were surprising because a dose of $25 \mu \mathrm{g}$ acetamiprid $\left(50 \mathrm{mg} \mathrm{L}^{-1}\right)$ caused similar mealworm mortality resulting from a combination of spores with the insecticide accumulated in the amount of 140 ng, i.e., almost 180-fold less. The difference in $\mathrm{LT}_{50}$ was one day, and for the highest dose of acetamiprid this value was determined on the fifth day of testing, and for the combination of spores and insecticide on day six. Acetamiprid at the highest dose acted slightly faster, while at the end of the experiment it turned out that the highest mortality was achieved for the combined action 
of spores and acetamiprid. Similarly to chemical insecticides, entomopathogens do not kill insects immediately and their lethal effect is delayed even up to 14 days [13]. When a combination of spores and acetamiprid was used, an effect similar to that achieved by the insecticide applied alone was observed. This suggests that when using a combination of spores and acetamiprid, the mechanism of action of the insecticide is can be followed, but due to the accumulation of acetamiprid in the spores, the form of application of the toxic compound has been changed, and therefore, it could be used in a smaller amount than in a situation when it is applied as the only insect-killing agent.

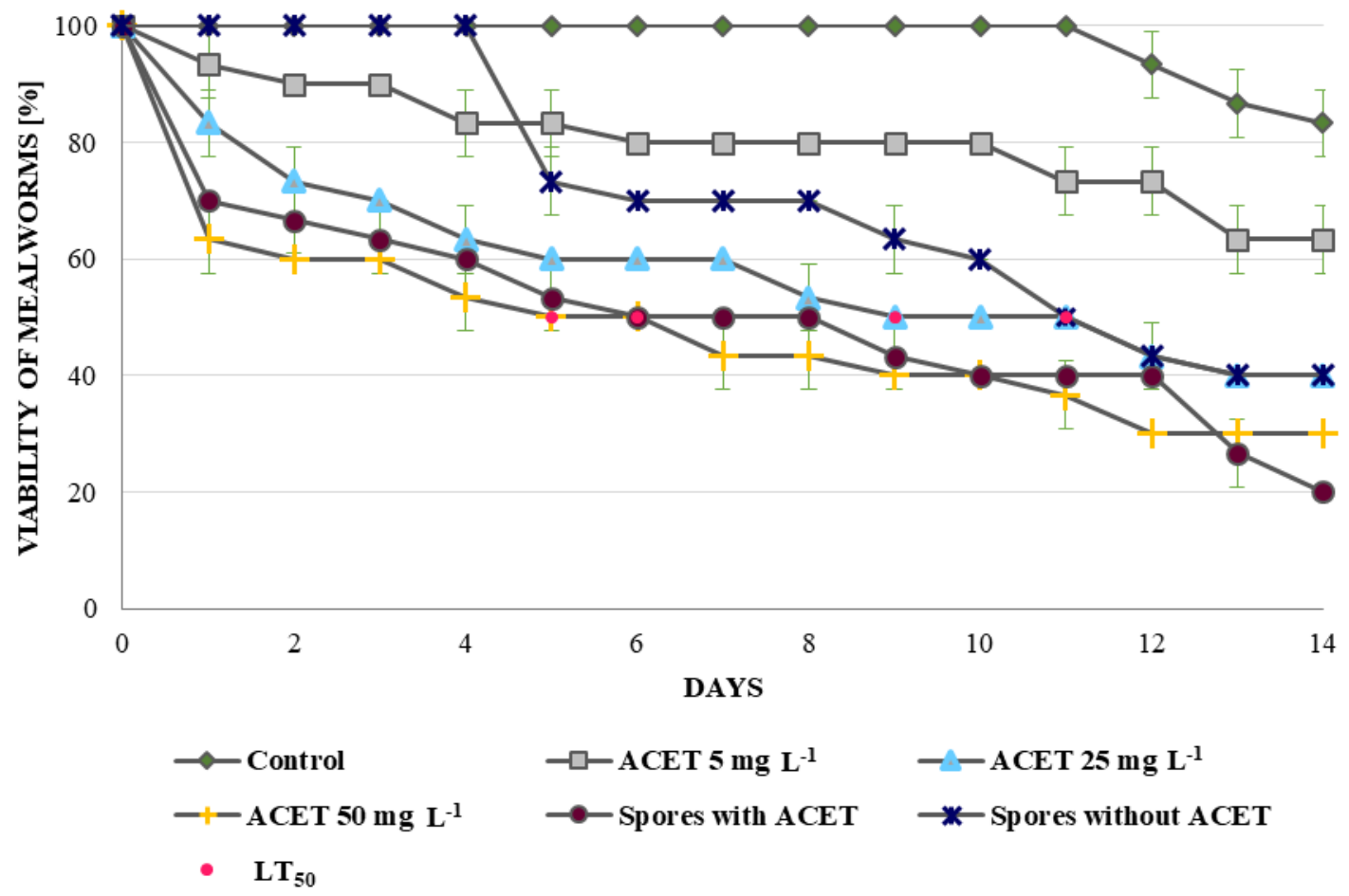

Figure 5. Viability of Tenebrio molitor mealworms treated with acetamiprid (ACET) at the concentrations of 5, 25 and $50 \mathrm{mg} \mathrm{L}^{-1}$, with Metarhizium brunneum spores and a combination of spores and ACET. Statistical significance was assessed by standard deviations.

In the natural environment, acetamiprid can impair dtxs production, thereby affecting the functioning of Metarhizium sp. in their habitat and the fight for an ecological niche. Due to the increased infectivity of Metarhizium spores applied with acetamiprid, it is possible that not only pests, but also beneficial arthropod species may be exposed to this toxic action.

\section{Conclusions}

The study reveals that, although acetamiprid does not inhibit the growth of Metarhizium sp., it affects the metabolism of the fungus by decreasing its ability to produce dtxs. This phenomenon may have environmental implications because dtxs are produced not only during infection but can also be important for the survival of Metarhizium in soil. It has been proved for the first time that acetamiprid accumulates in fungi, not only in plant and animal tissues, which could have some ecological implications as well. However, in the case of Metarhizium, the most important finding is the fact that acetamiprid increases its infectivity as in the experiments the spores with the accumulated insecticide were shown to cause the highest mortality of the tested larvae. On the other hand, in the light of the results obtained, it cannot be conclusively stated that a decrease in the production of dtxs caused by acetamiprid does not disturb the infection process because acetamiprid is a strong insecticide whose combined action with M. brunneum was revealed to be the most effective in the mortality tests of T. molitor. 


\section{Materials and Methods}

\subsection{Chemicals and Reagents}

All reagents and solvents were of analytical or liquid chromatography-mass spectrometry (LC-MS) grade and were purchased from Sigma-Aldrich (Steinheim, Germany) unless otherwise stated. Destruxin A (dtx A) from M. anisopliae and destruxin B (dtx B; Cayman Chemical, Ann Arbor, MI, USA) were used as chromatography standards. LC-MS grade water (Merck, Darmstadt, Germany) was used for chromatography. Fungi were cultivated on Czapek Dox broth (BD-Difco, Le Pont-de-Claix, France). Acetamiprid (99\% purity) was added to the fungal cultures and was also used as a chromatography standard.

\subsection{Microorganisms and Cultivation Conditions}

Six fungal strains of the genus Metarhizium (M. robertsii IM6519, M. robertsii IM2358, M. robertsii ARSEF727, M. anisopliae ARSEF7487, M. brunneum ARSEF2107, M. globosum ARSEF2596) from the strains collection of the ARSEF (The Agricultural Research Service Collection of Entomopathogenic Fungal Cultures) and the Department of Industrial Microbiology and Biotechnology, University of Lodz (Poland), were used during the investigations. All strains were maintained on ZT agar slants(glucose $\left(4 \mathrm{~g} \mathrm{~L}^{-1}\right)$; Difco yeast extract $\left(4 \mathrm{~g} \mathrm{~L}^{-1}\right)$; agar $\left(25 \mathrm{~g} \mathrm{~L}^{-1}\right)$; and malt extract $\left(6^{\circ} \mathrm{Blg}\right)$, up to $1 \mathrm{~L} ; \mathrm{pH}$ 7.0) as described previously [25-27]. Cultures for tests with Metarhizium spore suspension adjusted to $1 \times 10^{6}$ spores $\mathrm{mL}^{-1}$ were prepared on Czapek Dox liquid medium (total volume $40 \mathrm{~mL}$ in 100 mL Erlenmeyer flasks; BD-Difco, Le Pont-de-Claix, France). Acetamiprid stock dissolved in acetonitrile at the concentration of $50 \mathrm{mg} \mathrm{mL}^{-1}$ was added to fungal cultures to a final concentration of 5, 25 and $50 \mathrm{mg} \mathrm{L}^{-1}$. Additionally, abiotic controls (without fungal biomass) and biotic controls (without acetamiprid) were prepared. All samples were incubated on a rotary shaker (120 rpm) at $28^{\circ} \mathrm{C}$ for 7 days.

\subsection{Destruxins Extraction by a Modified QuEChERS Method}

After the incubation, the fungal cultures were filtrated through Whatman filter paper Number 1 (Sigma-Aldrich, Steinheim, Germany) to separate the culture media from the mycelia. The mycelia (for biomass estimation) were harvested and dried at $105^{\circ} \mathrm{C}$ until a constant weight was obtained. The culture media were centrifuged for $5 \mathrm{~min}$ at 10,000 rpm, and subsequently, $20 \mathrm{~mL}$ was transferred to each $50 \mathrm{~mL}$ Falcon tube with $10 \mathrm{~mL}$ acetonitrile. After vigorous vortexing for $1 \mathrm{~min}, 3000 \mathrm{rpm}$, QuEChERS salts $\left(4 \mathrm{~g} \mathrm{MgSO}_{4} ; 1 \mathrm{~g} \mathrm{NaCl} ; 1 \mathrm{~g} \mathrm{C}_{6} \mathrm{H}_{5} \mathrm{Na}_{3} \mathrm{O}_{7} \cdot 2 \mathrm{H}_{2} \mathrm{O} ; 0.5 \mathrm{~g} \mathrm{C}_{6} \mathrm{H}_{6} \mathrm{Na}_{2} \mathrm{O}_{7} \cdot 1.5 \mathrm{H}_{2} \mathrm{O}\right.$ ) were added and the tubes were again vortexed for $1 \mathrm{~min}$. Afterwards, the tubes were centrifuged for $5 \mathrm{~min}$ at $8000 \mathrm{rpm}$. Eight milliliters of the upper layer was transferred to each $15 \mathrm{~mL}$ Falcon tube and evaporated to dryness under reduced pressure at $40{ }^{\circ} \mathrm{C}$. After evaporation, the samples were dissolved in $5 \mathrm{~mL}$ LC-MS grade water (Merck, Darmstadt, Germany) and $4 \mathrm{~mL}$ of the extracts were cleaned on the Solid Phase Extraction Column with octadecyl sorbent C18. Subsequently, $5 \mathrm{~mL}$ of acetonitrile was added to rinse the metabolites bound on the sorbent, and next, $4 \mathrm{~mL}$ of the extract was transferred to each $15 \mathrm{~mL}$ Falcon tube and again evaporated to dryness as described above. After evaporation, the samples were dissolved in $2.5 \mathrm{~mL}$ of LC-MS grade water and $1 \mathrm{~mL}$ was subjected to LC-MS/MS.

\subsection{Acetamiprid Extraction by a Modified QuEChERS Method}

The mycelia were separated from Czapek Dox medium by filtration through Whatman filter paper Number 1 (Sigma-Aldrich, Steinheim, Germany). Then, $20 \mathrm{~mL}$ of deionized water and $10 \mathrm{~mL}$ of acetonitrile were added to each mycelium sample and ultrasonic extraction was done (2 min, Am $36 \%$, pulse for $10 \mathrm{~s}$ ). Then, $10 \mathrm{~mL}$ of acetonitrile was added to the culture medium in the volume $20 \mathrm{~mL}$, and ultrasonic extraction was performed as mentioned above. The subsequent procedure was the same for both types of samples. The samples were transferred to each $50 \mathrm{~mL}$ Falcon tube and extracted with a Ball Mill (Retch MM400, Idar-Oberstein, Germany) for 5 min and at 25/s frequency. 
After homogenization, QuEChERS salts were added and the tubes were vortexed $3000 \mathrm{rpm}$ for $1 \mathrm{~min}$. Subsequently, the extracts were centrifuged for $5 \mathrm{~min}$ at $5000 \mathrm{rpm}$. Then, $1 \mathrm{~mL}$ of the top layer was collected for the LC-MS/MS analysis.

\subsection{Acetamiprid Extraction from M. brunneum Spores and Subcellular Fractions}

To determine the acetamiprid concentration in spores, 7-day-old fungal cultures were filtered through the nylon net. The filtrates were centrifuged for $10 \mathrm{~min}$ at 10,000 rpm, and after supernatant removal, $20 \mathrm{~mL}$ of deionized water was added to the spores' pellet. The number of spores were counted in the Thoma cell counting chamber. Then, $10 \mathrm{~mL}$ of acetonitrile was added and the QuEChERS procedure was done as described above.

For acetamiprid presence in cell fractions, fungal cultures were centrifuged (10 min at 10,000 rpm), and after supernatant removal, the precipitate was washed twice with $20 \mathrm{~mL}$ of deionized water [28]. Then, the mycelium was suspended in $20 \mathrm{~mL}$ of deionized water and ultrasonic extraction was performed ( $2 \mathrm{~min}$, Am 36\%, pulse for $10 \mathrm{~s}$ ). Mycelia disintegration was controlled by cell oscopic observations. After mycelia disruption, the samples were centrifuged for $10 \mathrm{~min}$ at $1200 \mathrm{rpm}$. The supernatant was separated from the precipitate with the cell wall fraction, then it was transferred into Eppendorf tubes and centrifuged for $20 \mathrm{~min}$ at 20,000 rpm at $4{ }^{\circ} \mathrm{C}$. Obtained precipitates were suspended in $20 \mathrm{~mL}$ of deionized water, then $10 \mathrm{~mL}$ of acetonitrile was added and the QuEChERS extraction procedure was performed as described above. The cell wall fraction precipitate was washed twice in $20 \mathrm{~mL}$ of deionized water by centrifugation for $10 \mathrm{~min}$ at $1200 \mathrm{rpm}$. Then, it was suspended in $20 \mathrm{~mL}$ of deionized water, $10 \mathrm{~mL}$ of acetonitrile was added and the extraction procedure was followed as described above.

\subsection{LC-MS/MS Quantitative and Qualitative Analyses of Destruxins}

Quantitative and qualitative analyses of dtxs were carried out by using LC-MS/MS (LC Agilent 1200 coupled with a tandem mass spectrometer, AB Sciex QTRAP 4500, Framingham, MA, USA). The separation was performed with a Kinetex C18 column maintained at $40{ }^{\circ} \mathrm{C}$. Water with $5 \mathrm{mM}$ ammonium formate (AF, Solvent $\mathrm{A}$ ) and methanol with $5 \mathrm{mM}$ ammonium formate (Wolvent B) were used as mobile phases at a flow rate of $0.5 \mathrm{~mL} \mathrm{~min}^{-1}$. The injection volume was $5 \mu \mathrm{L}$. The eluent gradient was conducted as follows: hold $90 \%$ A from 0 to $0.25 \mathrm{~min}$, linear increase from $90 \%$ A to $90 \%$ B to $2 \mathrm{~min}$, hold $90 \%$ B from 2 to $4 \mathrm{~min}$, reverse to the initial conditions from 4 to $4.1 \mathrm{~min}$, and maintained for column equilibration to $6.0 \mathrm{~min}$.

The detection of $\mathrm{dtxs}$ was conducted using MS/MS with an electrospray ion source (ESI) in the positive ionization scheduled multiple reaction monitoring (sMRM) scan mode. The MRM detection window was set to $25 \mathrm{~s}$. The optimized ESI parameters were as follows: CUR: 25; IS: $5000 \mathrm{~V}$; TEMP: $500{ }^{\circ} \mathrm{C}$; GS1: 50; GS2:50. MRM parameters of $19 \mathrm{dtxs}$ are presented in Table S2 [29]. Dtxs were detected in the culture medium of each of the tested strains. The quantitative analyses of $\mathrm{dtx} A$ and $\mathrm{dtx} B$ were carried out using standard curves in the linearity range $2.5-100 \mathrm{ng} \mathrm{mL}^{-1}(r=0.9997$ and $r=0.9999$, respectively). The levels of the other dtxs were determined based on chromatographic peak areas and compared between samples using principal component analysis (PCA).

\subsection{LC-MS/MS Quantitative Analyses of Acetamiprid}

Quantitative analyses of acetamiprid were carried out using LC-MS/MS (LC Agilent 1200 coupled with a tandem mass spectrometer, AB Sciex QTRAP 3200). The separation was performed with a Kinetex $\mathrm{C} 18$ column maintained at $40{ }^{\circ} \mathrm{C}$. Water with $5 \mathrm{mM}$ AF (solvent A) and acetonitrile with $5 \mathrm{mM}$ AF and $0.1 \%$ formic acid (FA, Solvent B) were used as mobile phases at a flow rate of $0.5 \mathrm{~mL} \mathrm{~min}^{-1}$. The injection volume was $10 \mu \mathrm{L}$. The eluent gradient was conducted as follows: hold $90 \% \mathrm{~A}$ from 0 to $0.25 \mathrm{~min}$, linear increase from $90 \% \mathrm{~A}$ to $90 \% \mathrm{~B}$ to $0.5 \mathrm{~min}$, hold $90 \% \mathrm{~B}$ from 0.5 to $4 \mathrm{~min}$, reverse to initial conditions from 4 to $4.1 \mathrm{~min}$, and maintained for column equilibration to $6.0 \mathrm{~min}$. 
The detection of acetamiprid was conducted using MS/MS with ESI in the positive ionization MRM scan mode $(223.2 / 126.1 \mathrm{~m} / \mathrm{z} ; 223.2 / 73.0 \mathrm{~m} / \mathrm{z})$. The optimized ESI parameters were as follows: CUR: 25; IS: $5500 \mathrm{~V}$; TEMP: $500{ }^{\circ} \mathrm{C}$; GS1: 50; GS2:60. The quantitation curve of acetamiprid was accomplished in the quadratic regression in the range $25-1000 \mathrm{ng} \mathrm{mL}^{-1}$ and $r=0.9999$.

\subsection{Permeability of the Cell Membranes}

The intention was to check whether acetamiprid simply interferes with the transport of dtxs from the fungal cell to the culture medium, or its action is connected with another mechanism. The procedure was performed according to the method described by Siewiera et al., 2015 [30] with some modifications. Briefly, $1 \mathrm{~mL}$ each of the control samples and the tested samples with acetamiprid concentrations of 5 , 25 and $50 \mathrm{mg} \mathrm{L}^{-1}$ was transferred into Eppendorf tubes and then centrifuged for $10 \mathrm{~min}$ at 12,000 rpm. The supernatant was removed and $1 \mathrm{~mL}$ of Phosphate Buffered Saline (PBS) and $2 \mu \mathrm{L}$ of propidium iodide (stock solution $0.1 \mathrm{mg} \mathrm{mL}^{-1}$ ) were added to the precipitate and the mixture was vortexed for $30 \mathrm{~s}$ at $3000 \mathrm{rpm}$. After incubation in the dark for $5 \mathrm{~min}$, the supernatant was removed. The mycelium was washed twice in PBS by centrifugation in the conditions described previously. Finally, the samples were suspended in $1 \mathrm{~mL}$ of PBS and propidium iodide fluorescence was measured at $\lambda_{\mathrm{ex}}=540$ and $\lambda_{\mathrm{em}}=630$ (FLUOstar Omega, BMG LABTECH, Ortenberg, Germany). The final results were presented as fluorescence intensity per mg of dry weight.

\subsection{Mortality Test of Larvae of Tenebrio molitor (Mealworm)}

Three concentrations of acetamiprid $\left(5,25\right.$ and $\left.50 \mathrm{mg} \mathrm{L}^{-1}\right)$ and Metarhizium sp. spores $\left(1 \times 10^{6}\right.$ spores $\left.\mathrm{mL}^{-1}\right)$ with and without accumulated acetamiprid were tested. Control samples without any stressful factors were also done. The effects of the individual variants were checked using 10 mealworms, kept in the dark in plastic boxes with holes in the lid, and the bottom was lined with tissue paper. Before starting the experiment, the larvae were fed with oat flakes, which eliminates the death of starvation. Insect vitality was assessed daily for 14 days.

\subsection{Data Analysis}

Fungal biomass estimation and quantitative analyses of acetamiprid were conducted in four repetitions, analyses of dtxs in six repetitions. Measurements of propidium iodide fluorescence were carried out in four repetitions. The mortality test of larvae of T. molitor was conducted in triplicates. Variabilities of samples were given as standard deviations $( \pm \mathrm{SD})$. The one-way analysis of variance (ANOVA) and the posthoc Tukey test were used for investigations of statistical significance, using the concentrations of acetamiprid as a factor on transformed data. Scores at $p<0.05$ were classified as significant. Statistica 13.1 (StatSoft, Tulsa, OK, USA) was used to analyze the data. The qualitative data of dtxs were submitted to PCA in orthogonal rotation and normalization using total area sums and Pareto scaling (Marker View Software 1.2.1., AB Sciex, Framingham, MA, USA).

\section{Patents}

Patent application No. P.434091 in the Patent Office of the Republic of Poland.

Supplementary Materials: The following are available online at http://www.mdpi.com/2072-6651/12/9/587/s1, Figure S1: Elimination of acetamiprid at the concentrations of 5 (A), 25 (B) and $50 \mathrm{mg} \mathrm{L}^{-1}$ (C) after 7 days of incubation by Metarhizium sp. considering the residual content in the culture medium and mycelium; Table S1: Effect of acetamiprid on the permeability of the Metarhizium brunneum ARSEF2107 fungal cell wall; Table S2: Multiple Reaction Monitoring (MRM) parameters for determining 19 types of destruxins.

Author Contributions: M.N.: Conceptualization, Investigation, Methodology, Writing-review and editing; J.M.: Investigation; P.B.: Methodology; S.R.: Investigation, Methodology, Funding acquisition, Writing-review and editing. All authors have read and agreed to the published version of the manuscript.

Funding: This work was supported by the National Science Center in Krakow (Poland), grant number UMO-2016/23/B/NZ9/00840. 
Conflicts of Interest: The authors declare no conflict of interest.

\section{References}

1. Han, W.; Tian, Y.; Shen, X. Human exposure to neonicotinoid insecticides and the evaluation of their potential toxicity: An overview. Chemosphere 2018, 192, 59-65. [CrossRef]

2. Bass, C.; Denholm, I.; Williamson, M.S.; Nauen, R. The global status of insect resistance to neonicotinoid insecticides. Pestic. Biochem. Physiol. 2015, 121, 78-87. [CrossRef] [PubMed]

3. EFSA (European Food Safety Authority). Conclusion on the peer review of the pesticide risk assessment of the active substance acetamiprid. EFSA J. 2016, 14, 26. [CrossRef]

4. Xiong, J.; Wang, Z.; Ma, X.; Li, H.; You, J. Occurrence and risk of neonicotinoid insecticides in surface water in a rapidly developing region: Application of polar organic chemical integrative samplers. Sci. Total Environ. 2019. [CrossRef] [PubMed]

5. Ihara, M. Neonicotinoids: Molecular mechanisms of action, insights into resistance and impact on pollinators. Curr. Opin. Insect Sci. 2018, 30, 86-92. [CrossRef] [PubMed]

6. Lovett, B.; St. Leger, R.J. Genetically engineering better fungal biopesticides. Pest Manag. Sci. 2018, 74, 781-789. [CrossRef]

7. Mondal, S.; Baksi, S.; Koris, A.; Vatai, G. Journey of enzymes in entomopathogenic fungi. Pac. Sci. Rev. A Nat. Sci. Eng. 2016. [CrossRef]

8. Wang, B.; Kang, Q.; Lu, Y.; Bai, L.; Wang, C. Unveiling the biosynthetic puzzle of destruxins in Metarhizium species. Proc. Natl. Acad. Sci. USA 2012, 109, 1287-1292. [CrossRef]

9. Donzelli, B.G.G.; Krasnoff, S.B. Molecular Genetics of Secondary Chemistry in Metarhizium Fungi. Adv. Genet. 2016. [CrossRef]

10. Pedras, M.S.C.; Irina Zaharia, L.I.; Ward, D.E. The destruxins: Synthesis, biosynthesis, biotransformation, and biological activity. Phytochemistry 2002, 59, 579-596. [CrossRef]

11. Wang, X.; Gong, X.; Li, P.; Lai, D.; Zhou, L.; Wang, X.; Gong, X.; Li, P.; Lai, D.; Zhou, L. Structural diversity and biological activities of cyclic depsipeptides from fungi. Molecules 2018, 23, 169. [CrossRef] [PubMed]

12. Liu, B.L.; Tzeng, Y.M. Development and applications of destruxins: A review. Biotechnol. Adv. 2012, 30, 1242-1254. [CrossRef] [PubMed]

13. Litwin, A.; Nowak, M.; Różalska, S. Entomopathogenic fungi: Unconventional applications. Rev. Environ. Sci. Biotechnol. 2020, 19, 23-42. [CrossRef]

14. Ríos-Moreno, A.; Garrido-Jurado, I.; Resquín-Romero, G.; Arroyo-Manzanares, N.; Arce, L.; Quesada-Moraga, E. Destruxin A production by Metarhizium brunneum strains during transient endophytic colonisation of Solanum tuberosum. Biocontrol Sci. Technol. 2016, 26, 1574-1585. [CrossRef]

15. Taibon, J.; Sturm, S.; Seger, C.; Strasser, H.; Stuppner, H. Quantitative assessment of destruxins from strawberry and maize in the lower parts per billion range: Combination of a QuEChERS-based extraction protocol with a fast and selective UHPLC-QTOF-MS assay. J. Agric. Food Chem. 2015, 63, 5707-5713. [CrossRef]

16. Neves, P.M.O.J.; Hirose, E.; Tchujo, P.T.; Moino, J.R.A. Compatibility of entomopathogenic fungi with neonicotinoid insecticides. Neotrop. Entomol. 2001, 30, 263-268. [CrossRef]

17. Li, Y.; Long, L.; Yan, H.; Ge, J.; Cheng, J.; Ren, L.; Yu, X. Comparison of uptake, translocation and accumulation of several neonicotinoids in komatsuna (Brassica rapa var. perviridis) from contaminated soils. Chemosphere 2018, 200, 603-611. [CrossRef] [PubMed]

18. De Laet, C.; Matringe, T.; Petit, E.; Grison, C. Eichhornia crassipes: A Powerful Bio-indicator for Water Pollution by Emerging Pollutants. Sci. Rep. 2019, 9, 7326. [CrossRef]

19. Barbieri, M.V.; Postigo, C.; Guillem-Argiles, N.; Monllor-Alcaraz, L.S.; Simionato, J.I.; Stella, E.; Barceló, D.; López de Alda, M. Analysis of 52 pesticides in fresh fish muscle by QuEChERS extraction followed by LC-MS/MS determination. Sci. Total Environ. 2019, 653, 958-967. [CrossRef]

20. Bartlett, A.J.; Hedges, A.M.; Intini, K.D.; Brown, L.R.; Maisonneuve, F.J.; Robinson, S.A.; Gillis, P.L.; de Solla, S.R. Acute and chronic toxicity of neonicotinoid and butenolide insecticides to the freshwater amphipod, Hyalella azteca. Ecotoxicol. Environ. Saf. 2019, 175, 215-223. [CrossRef] 
21. Dong, T.; Zhang, B.; Weng, Q.; Hu, Q. The production relationship of destruxins and blastospores of Metarhizium anisopliae with virulence against Plutella xylostella. J. Integr. Agric. 2016, 15, 1313-1320. [CrossRef]

22. Ríos-Moreno, A.; Carpio, A.; Garrido-Jurado, I.; Arroyo-Manzanares, N.; Lozano-Tovar, M.D.; Arce, L.; Gámiz-Gracia, L.; García-Campaña, A.M.; Quesada-Moraga, E. Production of destruxins by Metarhizium strains under different stress conditions and their detection by using UHPLC-MS/MS. Biocontrol Sci. Technol. 2016, 26, 1298-1311. [CrossRef]

23. Ravindran, K.; Akutse, S.; Sivaramakrishnan, S.; Wang, L. Determination and characterization of destruxin production in Metarhizium anisopliae Tk6 and formulations for Aedes aegypti mosquitoes control at the field level. Toxicon 2016. [CrossRef] [PubMed]

24. Bernat, P.; Nykiel-Szymańska, J.; Stolarek, P.; Słaba, M.; Szewczyk, R.; Ró, S. 2,4-dichlorophenoxyacetic acid-induced oxidative stress: Metabolome and membrane modifications in Umbelopsis isabellina, a herbicide degrader. PLoS ONE 2018, 13, e0199677. [CrossRef]

25. Różalska, S.; Pawłowska, J.; Wrzosek, M.; Tkaczuk, C.; Długoński, J. Utilization of 4-n-nonylphenol by Metarhizium sp. isolates. Acta Biochim. Pol. 2013, 60, 677-682. [CrossRef]

26. Rózalska, S.; Glińska, S.; Długoński, J. Metarhizium robertsii morphological flexibility during nonylphenol removal. Int. Biodeterior. Biodegrad. 2014, 95, 285-293. [CrossRef]

27. Nowak, M.; Soboń, A.; Litwin, A.; Różalska, S. 4-n-nonylphenol degradation by the genus Metarhizium with cytochrome P450 involvement. Chemosphere 2019, 220, 324-334. [CrossRef]

28. Słaba, M.; Szewczyk, R.; Bernat, P.; Długoński, J. Simultaneous toxic action of zinc and alachlor resulted in enhancement of zinc uptake by the filamentous fungus Paecilomyces marquandii. Sci. Total Environ. 2009, 407, 4127-4133. [CrossRef]

29. Arroyo-Manzanares, N.; Diana Di Mavungu, J.; Garrido-Jurado, I.; Arce, L.; Vanhaecke, L.; Quesada-Moraga, E.; De Saeger, S. Analytical strategy for determination of known and unknown destruxins using hybrid quadrupole-Orbitrap high-resolution mass spectrometry. Anal. Bioanal. Chem. 2017, 409, 3347-3357. [CrossRef]

30. Siewiera, P.; Bernat, P.; Rózalska, S.; Długoński, J. Estradiol improves tributyltin degradation by the filamentous fungus Metarhizium robertsii. Int. Biodeterior. Biodegrad. 2015, 104, 258-263. [CrossRef] 